\section{ORIGINAL RESEARCH}

J.R. Petrella

S.E. Prince

S. Krishnan

H. Husn

L. Kelley

P.M. Doraiswamy

\title{
Effects of Donepezil on Cortical Activation in Mild Cognitive Impairment: A Pilot Double-Blind Placebo-Controlled Trial Using Functional MR Imaging
}

BACKGROUND AND PURPOSE: Cholinesterase-inhibitor therapy is approved for treatment of Alzheimer disease; however, application in patients with mild cognitive impairment $(\mathrm{MCl})$ is still under active investigation. The purpose of this study was to determine the effect of such therapy on the neural substrates underlying memory processing in subjects with $\mathrm{MCl}$ by using functional MR imaging (fMRI).

MATERIALS AND METHODS: Thirteen subjects with $\mathrm{MCl}$ (mean age, $68 \pm 6.9$ years) enrolled in a multicenter double-blind placebo-controlled trial testing the clinical efficacy of the cholinesteraseinhibitor, donepezil, were studied with fMRI at baseline and following 12 or 24 weeks of therapy (single-site pilot study). The cognitive paradigm was delayed-response visual memory for novel faces. Within-group 1-sample $t$ tests were performed on the donepezil and placebo groups at baseline and at follow-up. A repeated-measures analysis of variance design was used to look for a Treatment Group $\times$ Time interaction showing a significant donepezil- but not placebo-related change in blood oxygen level-dependent response during the course of the study.

RESULTS: At baseline, both groups showed multiple areas of activation, including the bilateral dorsolateral prefrontal cortex, fusiform gyrus, and anterior cingulate cortex. On follow-up, the placebo group demonstrated a decreased extent of dorsolateral prefrontal activation, whereas the donepezil group demonstrated an increased extent of activation in the ventrolateral prefrontal cortex. Interaction demonstrated significant donepezil- but not placebo-related change in the left inferior frontal gyrus.

CoNCLUSIONS: Despite the limitations inherent to a pilot study of a small sample, our results point to specific cortical substrates underlying the actions of donepezil, which can be tested in future studies.

$\mathbf{P}$ revious functional MR imaging (fMRI) studies have demonstrated that altered activation patterns in memory-specific brain regions may be a surrogate marker of neural dysfunction in patients with early-stage dementia or in an at-risk groups, such as those with mild cognitive impairment (MCI). ${ }^{1-5}$ Such a marker may be of benefit in clinical trials of cognitive-enhancing drugs, in which current clinical outcome measures are relatively hampered by measurement error and variability of change with time. ${ }^{6}$ For example, cognitive measurements are significantly affected by factors such as the patient's background and education as well as the tester's skills.

Received August 14, 2008; accepted after revision September 10.

From the Department of Radiology (J.R.P., S.E.P., S.K.), Alzheimer's Disease Imaging Research Laboratory and Brain Imaging and Analysis Center, and Departments of Psychiatry and Medicine (Geriatrics) (H.H., L.K., P.M.D.) and Center for Study of Aging (P.M.D.), Duke University Medical Center, Duke University, Durham, NC.

The study sponsor, Eisai/Pfizer, designed the overall multicenter clinical trial but did not have any role in the analyses or interpretation of the add-on fMRI study besides providing a grant to support it. Drs Petrella and Prince had access to all fMRI data and performed all fMRI acquisition and analyses. Dr Doraiswamy had access to all clinical data.

This study was supported by a grant from Eisai/Pfizer. Dr Doraiswamy has received research grants and honoraria from speaking or consulting from several pharmaceutical companies, including manufacturers of Alzheimer diagnostics and treatments. He owns stock in Sonexa Therapeutics. Dr Petrella has received research support from Eisai/Pfizer. Data previously presented in part at: International Conference on Alzheimer Disease, July 26-31, 2008, Chicago, III.

Please address correspondence to Jeffrey R. Petrella, MD, Department of Radiology, Alzheimer's Disease Imaging Research Laboratory and Brain Imaging and Analysis Center, Duke University Medical Center, Duke University, Durham, NC 27710; e-mail: petre001@ mc.duke.edu

DOI 10.3174/ajnr.A1359
In addition, such markers may serve as a predictor of beneficial clinical response in individual patients, not all of whom benefit from such agents.

Donepezil is the most widely used symptomatic therapy for Alzheimer disease $(\mathrm{AD})$ and is approved for treating mild, moderate, and severe stages of $\mathrm{AD}$. It is a selective acetylcholinesterase inhibitor that enhances the availability of acetylcholine in brain synapses. This mechanism is thought to underlie its cognitive effects because both $\mathrm{AD}$ and normal aging have been associated with loss of cortical cholinergic input from the nucleus basalis of Meynert. Donepezil is an investigational agent in MCI, and prior trials of this condition have been mixed. For example, in a 3-year trial testing whether donepezil could delay diagnosis of $\mathrm{AD}$ in patients with $\mathrm{MCI}$, its effects were positive during the entire trial only in a subgroup who carried the ApoE4 allele. ${ }^{7}$ Prior trials of other cholinergic agents in MCI, such as rivastigmine and galantamine, have shown negative results on their primary cognitive outcome measures. Despite donepezil not being an approved therapy for MCI, it is used in clinical practice as an off-label therapy, emphasizing the need for further research into both mechanisms and risk-benefits.

In this pilot study, we used a double-blind placebo-controlled design and a previously validated fMRI paradigm to study the patterns of cortical activation in subjects with MCI at baseline and following 12 and 24 weeks of therapy with donepezil. The purpose of this study was to determine the effect of cholinesterase-inhibitor therapy on the neural substrates underlying memory processing in subjects with MCI. 


\begin{tabular}{lccccc}
\hline \multicolumn{3}{l}{ Characteristics of subjects* } \\
\hline Characteristics & Overall & \multicolumn{2}{c}{ Donepezil } & \multicolumn{2}{c}{ Placebo } \\
\hline No. & 13 & \multicolumn{2}{c}{6} & \multicolumn{2}{c}{7} \\
Age (yr) & $68(6.9)$ & $64.7(6.0)$ & \multicolumn{2}{c}{$70.9(6.7)$} \\
Education (yr) $\dagger$ & $16.2(2.3)$ & \multicolumn{2}{c}{$17.5(2.1)$} & \multicolumn{2}{c}{$15.1(2.0)$} \\
Scores & & Pre & Post & Pre & Post \\
MMSEł & $28.3(1.7)$ & $28.3(1.9)$ & $29.8(0.4)$ & $28.3(1.7)$ & $28(2.5)$ \\
ADAS-Cog§ & $25.0(5.4)$ & $23.3(3.5)$ & $20.6(5.5)$ & $26.5(6.5)$ & $22.8(6.5)$ \\
NYU Delayed & $6.7(3.0)$ & $7.33(1.0)$ & $8.0(2.8)$ & $6.14(4.1)$ & $6.7(2.8)$ \\
$\quad$ Recall & & & & & \\
fMRI task & $79.1(11.8)$ & $77.1(16.4)$ & $78.0(10)$ & $79.1(8.9)$ & $86.3(13.5)$ \\
(\% correct) & & & & \\
\hline
\end{tabular}

Note:-NYU indicates New York University; MMSE, Mini-Mental State Examination; ADAS-Cog, Alzheimer Disease Assessment Scale-Cognitive Subscale.

${ }^{*}$ All table cells list the mean and standard deviation.

t Education refers to period of formal schooling as reported by the patient

$\ddagger$ Trend $(P=.09)$ for Treatment Group $\times$ Time interaction.

$\S$ Significant $(P<.05)$ main effect of time across both treatment groups.

\section{Materials and Methods}

\section{Subjects}

The Duke University institutional review board approved the study. All subjects gave written informed consent, and the study was Health Insurance Portability and Accountability Act-compliant. Fifteen subjects with MCI (mean age, $68 \pm 6.9$ years) were enrolled in a multicenter double-blind placebo-controlled clinical trial testing the clinical efficacy of donepezil. MCI selection criteria and the overall results from the clinical trial have been reported. ${ }^{8}$ Final determination of cognitive status was made by a board-certified psychiatrist (P.M.D.) with expertise in geriatric psychiatry.

The current fMRI study was designed as a single-site add-on to the clinical trial. fMRI scans were obtained at baseline and after 12 and 24 weeks of donepezil therapy, along with the cognitive assessments. All subjects were screened to rule out significant neuropsychiatric disorders, including dementia and depression, and subjects were randomly allocated to either the donepezil or placebo group. Selection and dosing criteria were as specified for the trial. ${ }^{8}$ Neuropsychological assessment consisted of the following tests: Clinical Dementia Rating, MiniMental State Examination (MMSE), Alzheimer Disease Assessment Scale-Cognitive Subscale (ADAS-Cog), New York University Paragraph Test Delayed Recall, Verbal Fluency, Boston Naming Test, Digit Symbol, Digit Span Backward, and Number Cancellation.

As such, this was a pilot study, and the sample size was determined by the logistics of recruitment as well as by prior positron-emission tomography and fMRI studies of drug effects. ${ }^{9}$ Although we originally proposed to determine activation in the prefrontal cortex and parietal and temporal lobes, we decided to examine the entire brain by using an exploratory approach, because the main goal of the study was to determine if donepezil effects could be detected by using fMRI in a controlled study and to provide hypotheses for future testing. Summary statistics for the sample are reported in the Table. Betweengroup unpaired Student $t$ tests on the baseline data and repeatedmeasures analysis of variance (ANOVA) Treatment Group $\times$ Time interaction were assessed for all clinical variables, as well as for the fMRI cognitive task.

\section{fMRI Cognitive Task}

The cognitive paradigm consisted of an event-related delayed-response visual memory task for novel faces and has been previously described in detail. ${ }^{10,11}$ Briefly, during the encoding phase, subjects were shown either 1 or 3 faces for 3 seconds, then were instructed to fixate on a crosshair for 15 seconds, followed by a retrieval phase in which a single face (probe) was presented for 1.5 seconds. The probe either matched or did not match a face shown during the immediately preceding encoding phase. In half of the trials, the probe stimulus matched 1 of the items in the memory array, whereas in the remaining half, the probe was a new stimulus. The subjects were instructed to respond as to whether the face matched or did not match. Six sets of the encoding/retrieval phases were shown in each run, and a total of 6 runs was performed, which resulted in a total of 36 trials with an overall imaging time of approximately 30 minutes for the functional acquisition. For the follow-up studies, different face stimuli were used to avoid practice effects; however, the stimuli were matched in difficulty with the first set.

All stimuli were presented by using a liquid crystal display projector (XGA Resolution, 900 lumens) equipped with a specially designed lens (Buhl Industries, Hamden, Ct). Stimuli were projected upon a 10 -inch-wide screen located within the magnet bore directly behind the subject's head, and subjects viewed the stimuli through mirrored glasses. Behavioral responses were monitored by using a button box incorporating a fiber-optic loop connected to a transistor-transistor logic driver circuit located outside the magnet room.

\section{Imaging}

All scanning was performed on a 1.5T NVi scanner (GE Healthcare, Milwaukee, Wis), equipped with an Advanced Development Workstation for real-time echo-planar imaging (EPI). A scout series of T1-weighted images was obtained in the sagittal plane (2D spin-echo; TR/TE, 500/10 ms; $256 \times 192$; FOV, 24 cm; 5.0-mm-thick/2.5 spacing), followed by axial dual-echo fast spin-echo images (TR/TE, 2700/ 20/80 ms; $256 \times 192$; FOV, 24 cm; 5.0-mm-thick/0.0 spacing), obtained for screening purposes. A board-certified neuroradiologist (J.R.P.) evaluated all anatomic scans clinically, screening for mass lesions or other clinically significant findings. The anterior and posterior commissures were identified in the midsagittal section of the initial scout series, and high-resolution coronal oblique anatomic images were acquired from 60 contiguous 5 -mm-thick coronal sections by using a $3 \mathrm{D}$ spoiled gradient-recalled $(\mathrm{SPGR})$ sequence $(\mathrm{TR} / \mathrm{TE}=$ $6 / \mathrm{min} ; 256 \times 256$; FOV, $24 \mathrm{~cm}$; flip angle, $25^{\circ} ; 5.0 \mathrm{~mm} / 0.0$ spacing). A time series of contiguous coronal oblique images sensitive to blood oxygen level-dependent (BOLD) contrast (gradient-echo EPI; TR/ TE, 3000/40 ms; 24-cm FOV; $64 \times 64$ image matrix; $90^{\circ}$ flip angle; section thickness, $5.0 \mathrm{~mm}$; gap, $0.0 \mathrm{~mm}$ ) was acquired from 32 contiguous coronal section locations chosen from the 3D SPGR series during performance of the cognitive activation task. A total of 98 brain volumes were obtained over each of the 6 runs described in the previous section.

\section{Image Analysis}

Only baseline and 24-week scans were included in the analysis, whenever possible. For those subjects unable to complete a 24 -week scanning or having low-quality scans due to scan-acquisition errors or motion $>5 \mathrm{~mm}$ in any of 3 orthogonal directions, the 12-week scan was carried forward in its place (last observation carried forward [LOCF]) for analysis purposes. Images were processed by using event-related analysis with Statistical Parametric Mapping (SPM2, SPM5) software (Wellcome Centre for Neuroimaging, http://www. fil.ion.ucl.ac.uk/spm). With SPM2, preprocessing of each subject's data consisted of section timing and motion correction, normalization to the Montreal Neurologic Institute template, and spatial smoothing with an 8-mm Gaussian kernel. For the first-level analysis, the BOLD response, measured as a percentage of the signal-intensity- 

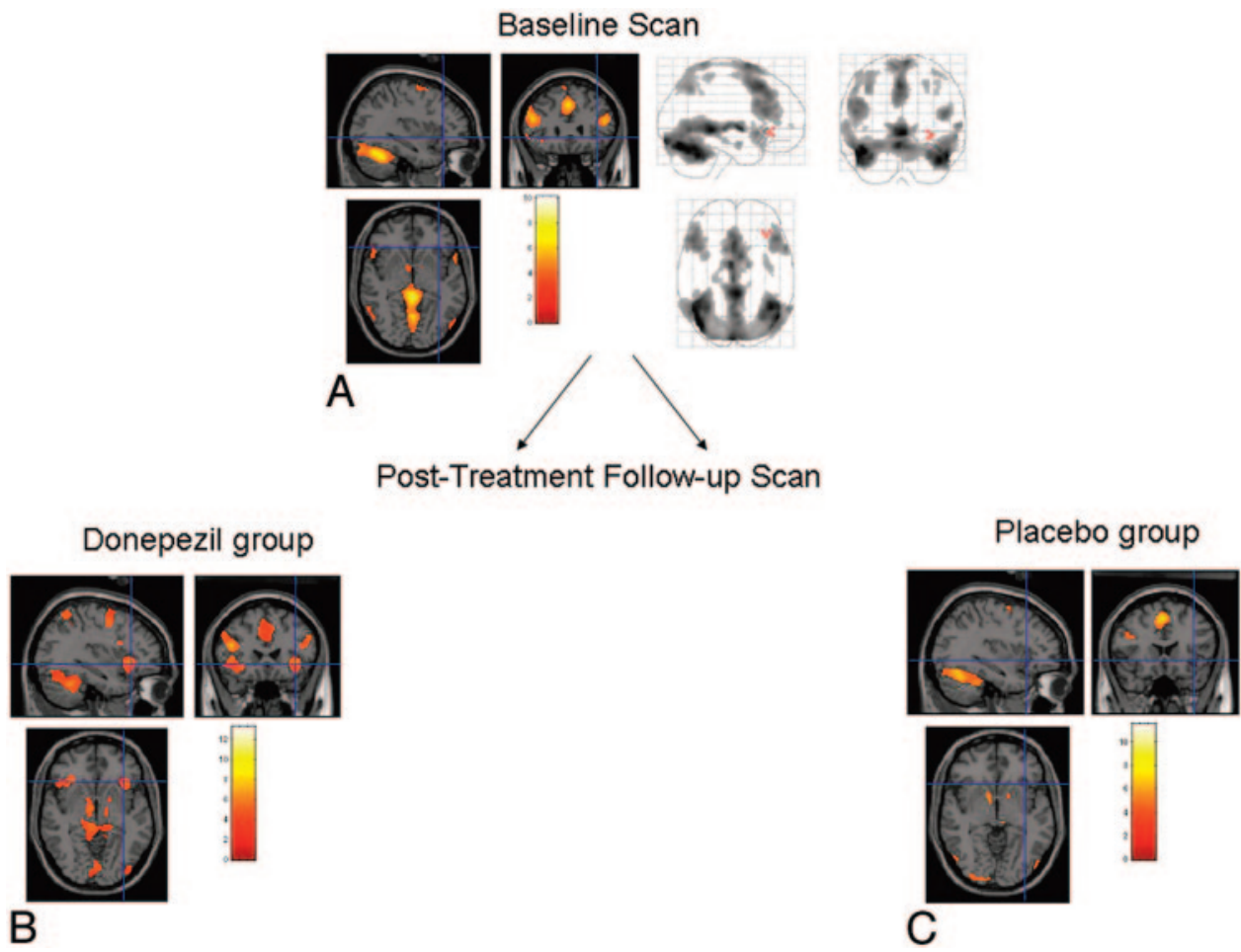

Fig 1. Statistical parametric maps of memory task versus baseline from 1-sample $t$ tests, pre- and posttreatment. All images are in radiologic orientation and are computed at a threshold of $P<.05$ false discovery rate-corrected, extent threshold of 10 voxels. $A, \mathrm{MCl}$ group at baseline. $B, \mathrm{MCl}$-donepezil group at follow-up scan demonstrates activation in the left inferior frontal gyrus as well as increased extent of activation in the ventrolateral prefrontal cortex, compared with baseline, and in both the bilateral dorso- and ventrolateral prefrontal cortex compared with the placebo group posttreatment. $C$, MCl-placebo group at follow-up scan does not demonstrate activation in left inferior frontal gyrus, but rather a decrease, compared with baseline, in the extent of dorsolateral prefrontal activation with relative preservation of anterior cingulate and fusiform activation.

change parameter estimate, was explicitly modeled on a voxel-wise basis for each encoding and retrieval event by using a general linear model; and a contrast map for encoding and retrieval was created for each subject. To maximize statistical power, we combined the encoding and retrieval contrast maps and performed the following secondlevel analyses by using SPM5:

1) Within-group 1 -sample $t$ test $(P<.05$, false discovery rate-corrected; 10-voxel extent threshold) of the entire group (both donepezil and placebo) at baseline and of the donepezil and placebo groups separately at follow-up.

2) Two-sample $t$ test $(P<.05$, false discovery rate-corrected; 10 -voxel extent threshold) of the donepezil and placebo groups at baseline.

3) Correlation $(P<.01$, uncorrected; 10-voxel extent threshold) of performance as measured by the ADAS-Cog score with the BOLD response in all subjects at baseline.

4) Two-sample repeated measures ANOVA design $(P<.05$, false discovery rate-corrected; 10-voxel extent threshold), in which the independent variables were treatment group and time. Here, the contrast of interest was the Treatment Group $\times$ Time interaction. This contrast was designed to identify clusters showing a significant donepezil- but not placebo-related change in the BOLD response during the course of the study. To restrict our search to brain regions of clinical significance for this paradigm in MCI, we applied the thresholded correlation map from the previous step as a mask to the ANOVA interaction contrast. The ADAS-Cog score was chosen because it is a common clinical outcome measure used in pharmaceutical trials of patients with dementia and MCI. ${ }^{6,8,9}$ Higher scores on this assessment signify greater levels of cognitive impairment.

\section{Results}

Of the 15 original subjects with MCI, 2 had incomplete imaging data from the baseline scan and were dropped from further analysis. Of the 13 subjects with MCI with a baseline and end point scan, 6 were in the donepezil group (mean age, $64.7 \pm$ 6.0 years; 6 males) and 7 were in the placebo group (mean age, $70.9 \pm 6.7$ years; 5 males). Mean scores for the cognitive tests and fMRI task are listed in Table 1 for both groups at baseline and posttreatment. In these 13 subjects, the end point scan used for outcome analysis was either the 24-week scan $(n=6$; 2 donepezil, 4 placebo) or 12 -week scan ( $n=7 ; 4$ donepezil, 3 placebo). No significant differences in baseline cognitive tests were demonstrated for any of the demographic or cognitive test variables. The ANOVA failed to demonstrate any significant Treatment Group $\times$ Time interaction for the cognitive test variables; however, there was a trend toward an interaction for improvement in MMSE in the donepezil compared with placebo group with time (denoted by a double dagger in the Table). There was a significant main effect in the ANOVA for Boston Naming, Digit Span Backward, and ADAS-Cog (denoted by a section mark in the Table), which showed improvement with repeat testing with time across both groups.

Within-group 1-sample $t$ tests at baseline and on follow-up are shown in Fig 1. At baseline, the MCI group showed multiple areas of activation, including the bilateral dorsolateral prefrontal cortex, fusiform gyrus, and anterior cingulate cortex (Fig 1A). There were no statistically significant differences in activation between the donepezil and placebo groups at baseline on the 2-sample $t$ test. Correlation between fMRI sig- 

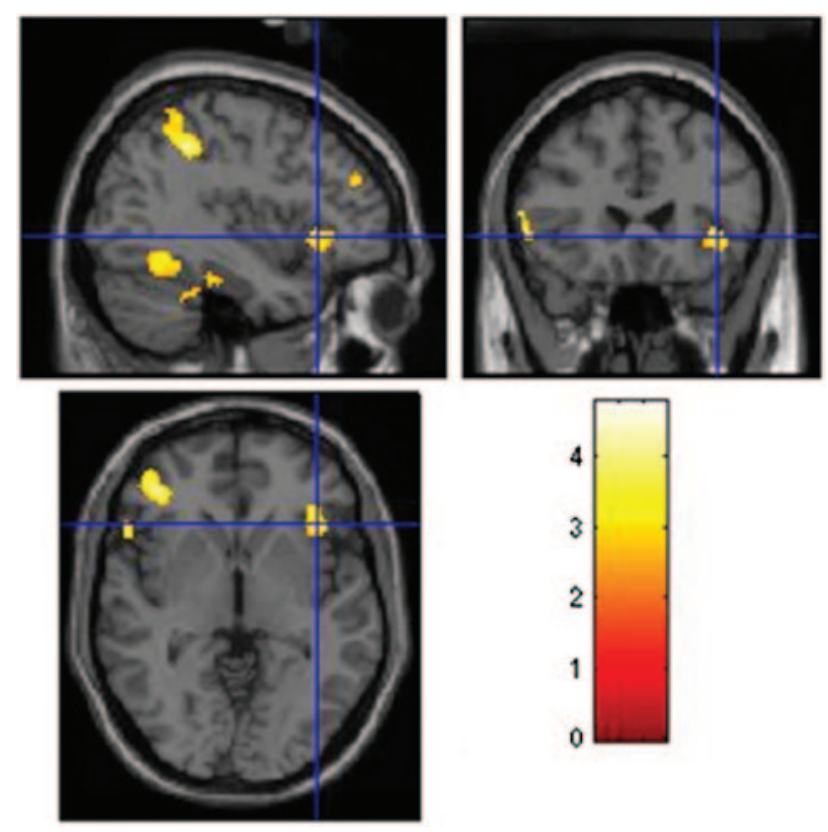

Fig 2. Regions displaying a negative correlation with ADAS-Cog $(P<.01$, uncorrected) in all $\mathrm{MCl}$ patients and at baseline, with the left inferior frontal gyrus shown at the crosshairs. Images are in radiologic orientation.

nal-intensity change and the ADAS-Cog score demonstrated significant foci of activation bilaterally in the frontal, lateral temporal, parietal, and fusiform gyrus regions (Fig 2). At the trial end point, the placebo group demonstrated a decrease, compared with baseline, in the extent of dorsolateral prefrontal activation with relative preservation of anterior cingulate and fusiform activation (Fig $1 C$ ). On the contrary, the donepezil group demonstrated increased extent in the ventrolateral prefrontal cortex, compared with baseline, and in both the bilateral dorso- and ventrolateral prefrontal cortices (Fig 1B), compared with the placebo group posttreatment.

Between-groups repeated-measure ANOVA Treatment Group $\times$ Time interaction revealed a single statistically significant cluster in the left inferior frontal gyrus (Talairach coordinates: -34, 27, - 1) (Fig 3A). Mean parameter estimates for fMRI BOLD response are depicted for the donepezil and placebo groups in the histogram (Fig 3B), as well as the correlation of BOLD response with baseline ADAS-Cog score (Fig $3 C$ ) within this cluster. Correlation analyses between cognitive improvement, measured by change in ADAS-Cog score, and either baseline or change in FMRI BOLD response within this cluster were not statistically significant.

\section{Discussion}

To our knowledge, this is the first double-blind placebo-controlled evaluation using fMRI to study the effect of a cholinesterase inhibitor on brain function in subjects with MCI. Our results suggest that donepezil, when administered during a 3to 6-month period to subjects with MCI, may potentially enhance brain activation in the left inferior frontal gyrus during memory processing.

The left inferior frontal gyrus has been implicated in an array of attention and memory processes, including encoding and retrieval and long- and short-term memory. ${ }^{12-16}$ Previous studies have shown this region to be implicated in subjects with MCI, compared with healthy elderly controls, during performance of memory tasks, including picture encoding. ${ }^{17}$ Moreover, initial studies of cholinergic-based drugs in AD or MCI have reported enhancement of functional activation levels in the frontal lobes, in general, as well as in the left inferior frontal gyrus. ${ }^{11,18-22}$ However, none of these studies were conducted in a double-blind fashion with a placebo control. Comparison with these and other fMRI studies of cholinergicbased drugs in MCI and AD is difficult because of differences in drug action (long-term-versus-short-term cholinesterase inhibition), trial design, cognitive task, and patient population.

The symptomatic efficacy of cholinergic drugs in $\mathrm{AD}$ is established, but their efficacy in MCI remains investigational. ${ }^{9,23}$ Lesion studies of cholinergic neurons in animals result in attention and, to a lesser extent, memory deficits, both of which are well described in early $\mathrm{AD}$; and these deficits in animal studies are reversed with cholinesterase inhibition. Hence, increased frontal lobe activation is a plausible underlying mechanism of response to cholinesterase inhibition. ${ }^{24}$

fMRI has potential benefit as an imaging marker of brain function in future trials of cognitive enhancing agents and may be of use in determining which patients with MCI best respond to therapy. Because $\mathrm{fMRI}$ requires active engagement in a cognitive task, it is uniquely suited for evaluation of cognitive-enhancing agents and may fit the requirements for a "mechanistic imaging" study in clinical trials of such agents. ${ }^{25}$

A number of imaging studies, including the current study, have demonstrated effects of cholinesterase inhibition on brain activation in sample sizes that were likely too small to demonstrate improvement in clinical outcome measures. One hypothesis raised is that of the superior sensitivity of imaging biomarkers such as fMRI in the evaluation of cognitive-enhancing drugs. On the other hand, the issue of whether these drug-induced imaging changes are replicable and cognitively beneficial is still not clear and would require that changes in activation in the affected brain areas correlate with changes in cognition or outcome. To our knowledge, this has only been demonstrated in the fMRI study by Saykin et al. ${ }^{20}$ Because the sample size in most imaging studies, including this pilot study, is necessarily small and the trial duration is short, there is very likely insufficient statistical power to demonstrate a significant cognitive benefit to do such a correlation. The time course of this study of 3-6 months is similar to that required for clinical efficacy of donepezil and other cholinesterase inhibitors ${ }^{26-29}$ and, therefore, more likely reflects a clinically relevant mechanism of action. Not surprisingly however, given the sample size, the neuropsychological measures in this study failed to demonstrate a statistically significant improvement in the donepezil compared with the placebo group, though there was a trend for improvement in the MMSE. In fact, as stated previously, the overall cognitive benefit of donepezil in MCI trials has been mixed, with the largest trial to date demonstrating benefits primarily in the ApoE4-positive subgroup during a 3-year period.

Limitations of this study include large interindividual variability in fMRI signal-intensity response and a small sample size. Intrinsic to $\mathrm{AMRI}$ is considerable variability in signal-intensity response at the level of the individual subject due to variability in task performance, baseline perfusion, and hemo- 

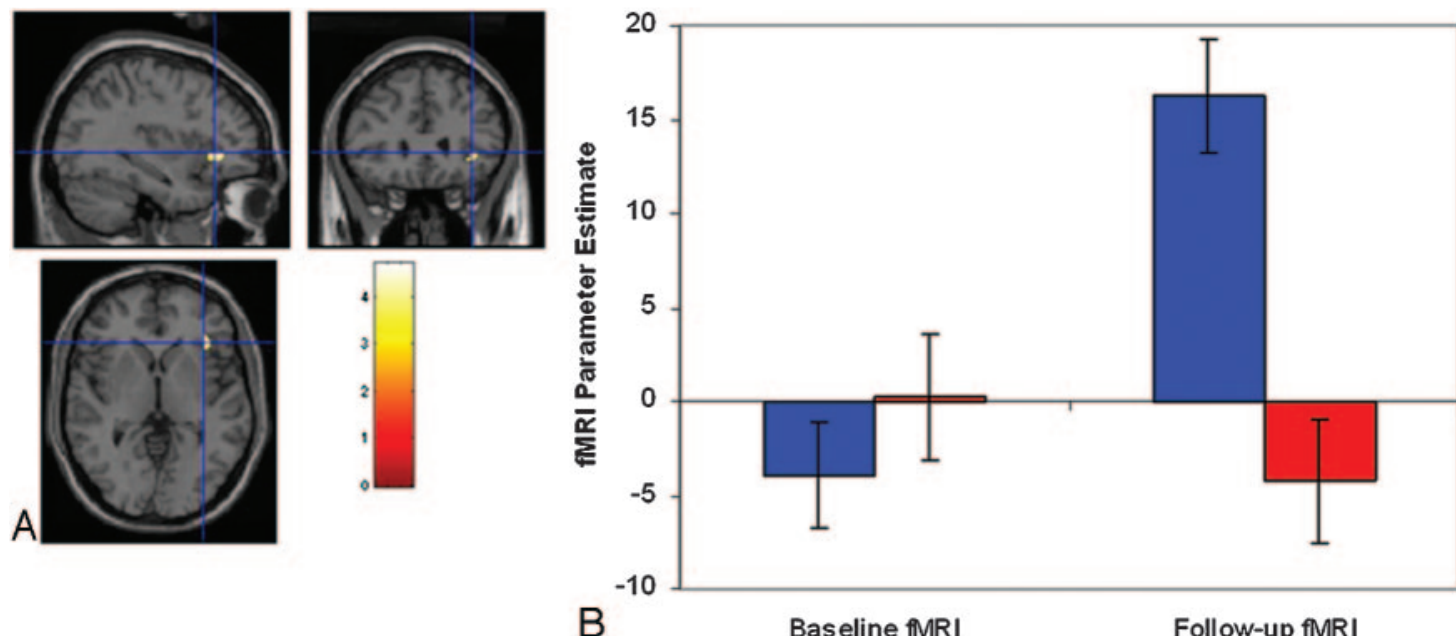

$\square$ Donepezil
$\square$ Placebo

B

Baseline fMRI

Follow-up fMRI

Bivariate Scattergram with Regression 95\% Confidence Bands

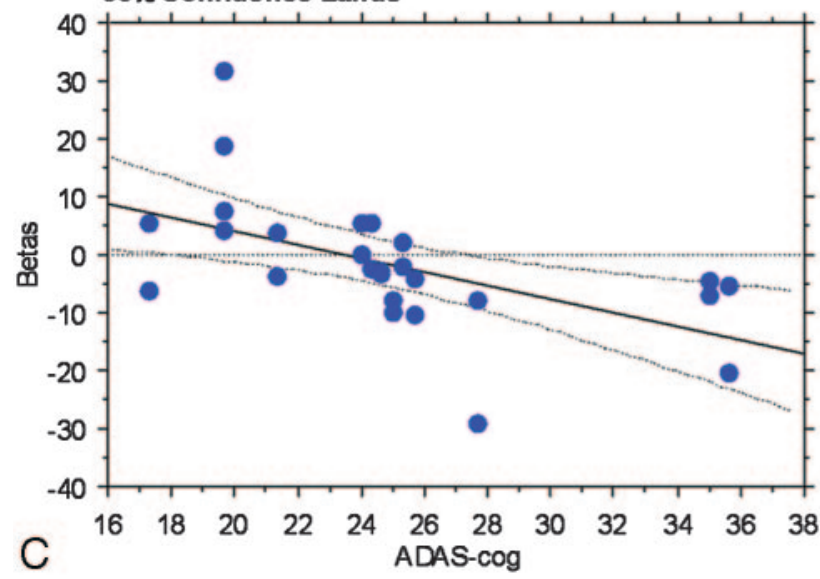

Fig 3. $A$, Area of the left inferior frontal gyrus shows significant Group $\times$ Time interaction $(P<.05$, corrected for false discovery rate) within regions showing significant correlation with the ADAS-Cog score ( $P<.01$ at baseline scan). Images are in radiologic orientation. $B$, Bar graph shows $\mathrm{fMRI}$ parameter estimate of activation magnitude ( \pm standard error). The Treatment Group $\times$ Time interaction is significant $(P<$.005). Post hoc $t$ tests reveal significant differences between follow-up and baseline fMRI for the donepezil $(P<.001)$ but not the placebo $(P=.45)$ group. There is no significant difference between donepezil and placebo at baseline $(P=.36)$ but a significant difference between donepezil and placebo at follow-up $(P<$ .001). $C$, Scatterplot shows significant negative correlation $(r=-0.55, P<.005)$ between the baseline fMRI parameter estimate (Betas) and the ADAS-Cog score across all subjects with $\mathrm{MCl}$. Higher scores on the ADAS-Cog signify greater levels of cognitive impairment.

dynamic response. ${ }^{30}$ Such factors are influenced by age and baseline cognition and may interact with conditions such as brain pathology and pharmacologic challenge, serving as confounders in pharmacologic fMRI studies. Because the number of subjects was small, we attempted to maximize statistical power at the level of the individual subject. Unlike most fMRI studies of memory, the encoding and retrieval conditions in the current study were combined rather than isolated in the analysis to maximize the number of events and, therefore, the signal-intensity-to-noise ratio at the level of the individual subjects. This could lead to some difficulty in interpretation of the results, though recent studies reveal a common network of nodes activated by both encoding and retrieval. ${ }^{31-34}$ The encoding and retrieval conditions in this study have been seen to individually and collectively activate a previously described network involving the fusiform gyrus and medial frontal and dorsolateral prefrontal cortices, in both young and elderly controls. $^{11,31-34}$

In addition, heterogeneity of clinical response to a drug is a well-known phenomenon in pharmaceutic trials, and future pharmacologic fMRI studies should incorporate this factor to increase power by separating analysis of patients who respond favorably from those who do not, because the underlying neural response may differ in both groups. Furthermore, the ability to characterize and predict such a differential clinical response may be of tremendous benefit in targeting individualized therapies. fMRI holds promise in this regard, particularly with respect to cognitive-enhancing therapies.

\section{Conclusions}

Despite the limitations inherent to a pilot study, our results identify enhancement of brain activation in the left inferior frontal gyrus as a possible neural response to donepezil therapy. Future studies to test whether such an imaging marker may serve as a surrogate for clinical response in trials of cognitive-enhancing pharmaceutic agents as well as a predictor of clinical response in individual patients are needed.

\section{References}

1. Drzezga A. Concept of functional imaging of memory decline in Alzheimer's disease. Methods 2008;44:304-14

2. Ries ML, Carlsson CM, Rowley HA, et al. Magnetic resonance imaging charac- 
terization of brain structure and function in mild cognitive impairment: a review. J Am Geriatr Soc 2008;56:920-34

3. Krishnan S, Talley BD, Slavin MJ, et al. Current status of functional MR imaging, perfusion-weighted imaging, and diffusion-tensor imaging in Alzheimer's disease diagnosis and research. Neuroimaging Clin N Am 2005; 15:853-68

4. Saykin AJ, Wishart HA. Mild cognitive impairment: conceptual issues and structural and functional brain correlates. Semin Clin Neuropsychiatry 2003;8:12-30

5. Petrella JR, Coleman RE, Doraiswamy PM. Neuroimaging and early diagnosis of Alzheimer disease: a look to the future. Radiology 2003;226:315-36

6. Dickerson BC, Sperling RA. Neuroimaging biomarkers for clinical trials of disease-modifying therapies in Alzheimer's disease. NeuroRx 2005;2:348-60

7. Petersen RC, Thomas RG, Grundman M, et al. Vitamin E and donepezil for the treatment of mild cognitive impairment. N Engl J Med 2005;352:2379-88

8. Salloway S, Ferris S, Kluger A, et al. Efficacy of donepezil in mild cognitive impairment: a randomized placebo-controlled trial. Neurology 2004;63: 651-57

9. Venneri A. Imaging treatment effects in Alzheimer's disease. Magn Reson Imaging 2007;25:953-68

10. Petrella JR, Lustig C, Bucher LA, et al. Prefrontal activation patterns in subjects at risk for Alzheimer disease. Am J Geriatr Psychiatry 2002;10:112-13

11. Petrella JR, Townsend BA, Jha AP, et al. Increasing memory load modulates regional brain activity in older adults as measured by fMRI. J Neuropsychiatry Clin Neurosci 2005; 17:75-83

12. Cabeza R, Nyberg L. Imaging cognition II: an empirical review of 275 PET and fMRI studies. J Cogn Neurosci 2000;12:1-47

13. Prince SE, Daselaar SM, Cabeza R. Neural correlates of relational memory: successful encoding and retrieval of semantic and perceptual associations. J Neurosci 2005;25:1203-10

14. Prince SE, Tsukiura T, Cabeza R. Distinguishing the neural correlates of episodic memory encoding and semantic memory retrieval. Psychol Sci 2007;18: $144-51$

15. Ranganath C, Johnson MK, D'Esposito M. Prefrontal activity associated with working memory and episodic long-term memory. Neuropsychologia 2003;41: $378-89$

16. Wagner AD, Pare-Blagoev EJ, Clark J, et al. Recovering meaning: left prefrontal cortex guides controlled semantic retrieval. Neuron 2001;31:329-38

17. Mandzia JL, McAndrews MP, Grady CL, et al. Neural correlates of incidental memory in mild cognitive impairment: an fMRI study. Neurobiol Aging 2007 Oct 24. [Epub ahead of print]

18. Rombouts SA, Barkhof F, Van Meel CS, et al. Alterations in brain activation during cholinergic enhancement with rivastigmine in Alzheimer's disease. J Neurol Neurosurg Psychiatry 2002;73:665-71

19. Goekoop R, Rombouts SA, Jonker C, et al. Challenging the cholinergic system in mild cognitive impairment: a pharmacological fMRI study. Neuroimage 2004;23:1450-59

20. Saykin AJ, Wishart HA, Rabin LA, et al. Cholinergic enhancement of frontal lobe activity in mild cognitive impairment. Brain 2004;127:1574-83

21. Bentley P, Driver J, Dolan RJ. Cholinesterase inhibition modulates visual and attentional brain responses in Alzheimer's disease and health. Brain 2008;131 409-24

22. Shanks MF, McGeown WJ, Forbes-McKay KE, et al. Regional brain activity after prolonged cholinergic enhancement in early Alzheimer's disease. Magn Reson Imaging 2007;25:848-59

23. Leyhe T, Stransky E, Eschweiler GW, et al. Increase of BDNF serum concentration during donepezil treatment of patients with early Alzheimer's disease. Eur Arch Psychiatry Clin Neurosci 2008;258:124-28

24. Mesulam M. The cholinergic lesion of Alzheimer's disease: pivotal factor or side show? Learn Mem 2004;11:43-49

25. Matthews B, Siemers ER, Mozley PD. Imaging-based measures of disease progression in clinical trials of disease-modifying drugs for Alzheimer disease. Am J Geriatr Psychiatry 2003;11:146-59

26. Tune L, Tiseo PJ, Ieni J, et al. Donepezil HCl (E2020) maintains functiona brain activity in patients with Alzheimer disease: results of a 24-week, doubleblind, placebo-controlled study. Am J Geriatr Psychiatry 2003;11:169-77

27. Knapp MJ, Knopman DS, Solomon PR, et al. A 30-week randomized controlled trial of high-dose tacrine in patients with Alzheimer's disease: The Tacrine Study Group. JAMA 1994;271:985-91

28. Rogers SL, Farlow MR, Doody RS, et al. A 24-week, double-blind, placebocontrolled trial of donepezil in patients with Alzheimer's disease: Donepezil Study Group. Neurology 1998;50:136-45

29. Tariot PN, Solomon PR, Morris JC, et al. A 5-month, randomized, placebocontrolled trial of galantamine in AD: The Galantamine USA-10 Study Group Neurology 2000;54:2269-76

30. Miller MB, Van Horn JD, Wolford GL, et al. Extensive individual differences in brain activations associated with episodic retrieval are reliable over time. Cogn Neurosci 2002;14:1200-14

31. Kahn I, Davachi L, Wagner AD. Functional-neuroanatomic correlates of recollection: implications for models of recognition memory. J Neurosci 2004;24:4172-80

32. Sperling RA, Bates JF, Cocchiarella AJ, et al. Encoding novel face-name associations: a functional MRI study. Hum Brain Mapp 2001;14:129-39

33. Leube DT, Erb M, Grodd W, et al. Successful episodic memory retrieval of newly learned faces activates a left fronto-parietal network. Brain Res Cogn Brain Res 2003;18:97-101

34. Grady CL, McIntosh AR, Bookstein F, et al. Age-related changes in regional cerebral blood flow during working memory for faces. Neuroimage 1998;8: 409-25 